but 3 out of 4 DOT1L inhibited samples showed a significantly reduced effect of TGF $\beta$ on ACTA2 expression. The amount of collagen I and III in the extracellular matrix after 72 hours of TGF $\beta$, was comparable between control and EPZ treated fibroblasts. BrdU labelling assay showed increased proliferation with DOT1L inhibition. In vivo, subcutaneous bleomycin induced an increased dermal thickness and skin collagen content in mice. No difference in the effect of bleomycin was found between mice with a conditional fibroblastspecific DOT1L knockout or wild type mice.

Conclusions In an in vitro model of fibrosis, primary human dermal fibroblasts treated with a DOT1L-inhibitor showed increased proliferation and reduced upregulation of ACTA2 but did not result in detectable differences in collagen deposition. In an in vivo murine model of skin fibrosis, no difference in bleomycin-induced skin thickness and collagen content was found when DOT1L was knocked out in fibroblasts.

Disclosure of interest None declared

\section{P102 S100A9 HAMPERS OSTEOCLAST DIFFERENTIATION FROM CIRCULATING PRECURSORS BY REDUCING THE EXPRESSION OF RANK}

${ }^{1} \mathrm{M}$ van den Bosch, ${ }^{1} \mathrm{I}$ Di Ceglie, ${ }^{2} \mathrm{~T}$ Vogl, ${ }^{2} \mathrm{~J}$ Roth, ${ }^{3} \mathrm{C}$ Goodyear, ${ }^{1} \mathrm{P}$ van der Kraan, ${ }^{1} \mathrm{~A}$ Blom, ${ }^{1} \mathrm{P}$ van Lent*. ${ }^{1}$ Radboud university medical centre, Nijmegen, Netherlands; ${ }^{2}$ University of Muenster, Muenster, Germany; ${ }^{3}$ University of Glasgow, Glasgow, UK

\subsection{6/annrheumdis-2018-EWRR2018.118}

Introduction The alarmins S100A8/A9 are produced in high levels in the synovium during both experimental and human rheumatoid arthritis (RA) and have been implicated in inflammation-induced bone resorption. We and others have previously shown that stimulation of mature osteoclasts with S100A8/A9 results in increased numbers and resorptive activity. In agreement, reduced bone destruction was observed after induction of experimental RA models in S100a $9^{-/-}$mice. However, the effects of S100A8/A9 on monocyte-to-osteoclast differentiation remain elusive.

Objectives Here, we investigated the effects of S100A9 on CD $14^{+}$monocytes and their potential to differentiate into osteoclasts.

Methods CD14 ${ }^{+}$monocytes were isolated from buffy coats of healthy donors using density gradient centrifugation and magnetic cell sorting. Cells were differentiated into osteoclasts with macrophage colony-stimulating factor (M-CSF) and Receptor activator of nuclear factor kappa-B (RANK) ligand (RANKL) in the presence or absence of S100A9. mRNA expression was determined by RT-qPCR and protein expression was determined using Luminex analysis. Moreover, osteoclast differentiation was assessed using Tartrate-resistant acid phosphatase (TRAP) staining and the resorptive capacity was determined using mineral-coated plates. RANK protein expression was assessed using FACS.

Results We observed that S100A9 stimulation of monocytes resulted in a strong induction of various pro-inflammatory factors, such as interleukin (IL)1 $\beta$, IL6, IL8, and tumour necrosis factor (TNF) $\alpha$ after 24 hour, both on the mRNA and protein level. Interestingly, we observed a strong decrease in the number of multinucleated osteoclasts as determined by TRAP staining, at day 6 and 8 after start of the cultures. In agreement with this, the cells showed a strongly reduced resorptive capacity. We demonstrated that already a 24 hour stimulation with S100A9 strongly reduced the osteoclastogenic potential of the $\mathrm{CD}^{+} 4^{+}$monocytes. Finally, we observed that S100A9 stimulation hampered the M-CSF-induced upregulation of RANK, which could be reversed by addition of the TNF $\alpha$ inhibitor etanercept, but not the interleukin 1 receptor antagonist.

Conclusions Whereas S100A8/A9 have been previously shown to stimulate the numbers and resorptive capacity of mature osteoclasts, we here show that stimulation of monocytes with S100A9 strongly inhibits their osteoclastogenic potential, possibly via $T N F \alpha$-induced reduction of RANK expression. This suggests that the timing of exposure to S100A8/A9 is an important determinant for monocyte-to-osteoclast differentiation.

Disclosure of interest None declared

\section{P103 PRO-FIBROTIC RESPONSES INDUCED BY THYMIC STROMAL LYMPHOPOETIN}

${ }^{1} \mathrm{~L}$ Wang, ${ }^{2} \mathrm{~S}$ Wang, ${ }^{3} \mathrm{~V}$ Taneja, ${ }^{2} \mathrm{R}$ Vassallo. 'Department of Pulmonary Medicine, Guang'anmen Hospital, China Academy of Chinese Medicine Science, Beijing, China; ${ }^{2}$ Pulmonary and Critical Care Medicine; ${ }^{3}$ Immunology, Mayo Clinic, Rochester, Minnesota, United States

\subsection{6/annrheumdis-2018-EWRR2018.119}

Registration status I still need to register to EWRR Introduction Thymic Stromal Lymphopoetin (TSLP) is a T helper-2 (Th2) cytokine that may be an important regulator of tissue fibrosis. ${ }^{1}$ Rheumatoid Arthritis (RA)-associated interstitial lung disease is a fibrotic extra-articular complication associated with dysregulated adaptive immunity in the lung. In a recent study - Vassallo et al. Clin Immunol 2014;152(1-2):25-35 we reported increased TSLP mRNA levels in the lungs of cigarette smoke-exposed, collagen induced arthritis mice with a DQ8 background. ${ }^{2}$

Objectives The purpose of this study was to explore direct molecular mechanisms by which TSLP induces fibrogenesis.

Methods We performed in vitro studies with human lung fibroblasts (Lonza) incubated with recombinant human TSLP (R\&D Systems, 0-10 ng/ml) and determined TSLP-induced proliferation using a CyQuant assay, as well as collagen and fibronectin gene expression. We also investigated signaling intermediates activated by TSLP utilizing a Signal Transduction and Activator of Transcription (STAT)1-6 ELISA assay on lysates generated from TSLP stimulated fibroblasts at varying time points.

Results Human lung fibroblasts proliferate directly in response to TSLP with concentrations $\geq 1 \mathrm{ng} / \mathrm{ml}$ inducing a significant proliferative response compared with controls. TSLP also induced fibronectin and collagen gene expression in lung fibroblasts. Incubation with TSLP induced phosphorylation of STAT-1 and STAT-3: phosphorylation of STAT-2, as well as 4-6 was not observed. Phosphorylation of STAT-1 and 3 were observed within 30 minutes of incubation with TSLP (10 ng/ $\mathrm{ml})$.

Conclusions These in vitro studies demonstrate that fibroblasts are directly responsive to recombinant human TSLP which induces proliferation and matrix generation, potentially through activation of STAT-1 and 3. These observations further support a role for TSLP as a regulator of tissue fibrosis in the lung, and suggest a potential therapeutic target for the treatment of autoimmunity related fibrosis. 


\section{REFERENCES}

1. Datta A, Alexander R, Sulikowski MG, Nicholson AG, Maher TM, Scotton CJ, Chambers RC. Evidence for a functional thymic stromal lymphopoietin signaling axis in fibrotic lung disease. J Immunol November 1 2013;191(9):4867-79.

2. Vassallo R, Luckey D, Behrens M, Madden B, Luthra H, David C, Taneja V. Cellular and humoral immunity in arthritis are profoundly influenced by the interaction between cigarette smoke effects and host HLA-DR and DQ genes. Clin Immunol May-June 2014;152(1-2):25-35.

Disclosure of interest L. Wang: None declared, S. Wang: None declared, V. Taneja : None declared, R. Vassallo Grant/research support from: Pfizer and Bristol Myers Squibb

\section{P104 SYNOVIAL FIBROBLAST RELATIONSHIP STATUS: IT'S COMPLICATED}

${ }^{1}$ RA Byrne*, ${ }^{1}$ I Olmos Calvo, ${ }^{2}$ F Kartnig, ${ }^{1}$ A Platzer, ${ }^{3} V$ Zheden, ${ }^{3} \mathrm{~L}$ Lovicar, ${ }^{4} \mathrm{~J}$ Holinka, ${ }^{1} \mathrm{G}$ Steiner, ${ }^{5} \mathrm{P}$ Ertl, ${ }^{1}$ JS Smolen, ${ }^{1} \mathrm{H}$-P Kiener. 'Dep. of Medicine III - Rheumatology, Medical University of Vienna; ${ }^{2}$ CEMM, Vienna; ${ }^{3}$ Institute for Science and Technology Austria, Klosterneuburg; ${ }^{4}$ Dep. of Orthopaedics, Medical University of Vienna; ${ }^{5}$ Dep. of Chemical Technologies and Analytics, TU Vienna, Vienna, Austria

\subsection{6/annrheumdis-2018-EWRR2018.120}

Introduction The synovium is primarily built by fibroblast-likesynoviocytes (FLS). In the healthy synovium FLS form a complex tissue network via long-distance intercellular connexions (nanotubes). Using a 3D synovial micromass culture system, our previous research demonstrated that FLS exchange cytoplasmic cargo, i.e. organelles like mitochondria, via transfer through direct cell-to-cell connexions. The adaptive synovial tissue response to inflammation (i.e. to TNFa) likely depends upon the concerted communication between FLS.

Objectives To determine how the cellular organisation of the synovial tissue affects intercellular cargo exchange and how it changes under inflammatory conditions.

Methods Human FLS were isolated from joint tissues after synovectomies; passaged FLS were used to generate 3D micromass cultures using Matrigel (BD). For ultrastructural 3D reconstruction ultrathin sections of fixed micromasses were cut with an ATUMtom for FE-SEM scanning, followed by alignment and reconstruction of individual cells with Fiji TrackEM. To compare unstimulated and TNF-stimulated micromass cultures, cells were dyed with Cell- and Mitotracker dyes (TF). Using confocal microscopy, micromass cultures were analysed for cellular organisation within the 3D sphere, cell volume of individual cells, cell-to-cell interconnectivity and cargo transfer between cells. Analyses of the 3D confocal imaging data were done in Bitplane Imaris.

Results SEM 3D reconstruction of synovial micromass tissue revealed that FLS are compartmentalised, and thus, much larger and extended than expected. Thin membrane tubes not only connect different cells but also compartments of the same cell. Additionally, one nanotube may provide a link to several other cells and these connexions are in general separated by membranes. Treatment of micromasses with TNFa resulted in cellular condensation, reduced cell volume as well as diminished cellular connectivitiy when compared to unstimulated micromasses. In particular, the abundance of interconnecting nanotubes was significantly attenuated. The mitochondrial transfer rate increased as FLS clustered upon TNFa stimulation.

Conclusions TNFa directs FLS cellular re-organisation that is associated with increased intercellular transfer of mitochondria. These studies may provide insight into the concerted cooperation of FLS that is likely critical for the adaptive synovial response to inflammation.

Disclosure of interest None declared

\section{P105 ENDOPLASMIC RETICULUM STRESS MEDIATES DERMAL FIBROSIS}

S O'reilly*. Life sciences, Northumbria University, Newcastle Upon Tyne, UK

10.1136/annrheumdis-2018-EWRR2018.121

Introduction Systemic sclerosis is an autoimmune idiopathic connective tissue disease. The cells mainly responsible are the myofibroblasts that secrete copious amount of extracellular matrix molecules such as collagen. Endoplasmi reticulum stress is induced by a variety of stressors including misfolded proteins, this leads to a classic stress response and activation of X Box Binding Protien-1 (XBP-1) and ATF-6. This is induced in a variety of situations from multiple stressors and is a conserved pathway. Its role in systemic sclerosis is unknown.

Objectives To examine the role of endoplasmic reticulum stress in systemic sclerosis.

Methods Healthy dermal fibroblasts were treated with the ER stress inducing agent thaspigargin or nothing and then lysed and various proteins such as XBP-1 and ATF measured along with collagen by western blotting and reprobed for a loading control. In some experiments small interfering RNA against XBP-1, a mediator of ER stress, was transfected and treated with thaspigargin or non targeting controls. Also the belomycin mouse model of fibrosis was used and ATF6 mRNA was measured using qRT-PCR.

Results The classic ER stress inducer thaspigargin induced collagen expression in dermal fibroblasts as well as the markers of ER stress XBP-1 and ATF-6. The ER stress induced collagen expression in dermal fibroblasts was reduced with the reduction of XBP-1 in these cells using small interfering RNA compared to non targeting siRNA. The bleomycin model of skin fibrosis revealed increased ATF-6 mRNA levels compared to vehicle control tissue.

Conclusions Endoplasmic reticulum stress appears to regulated collagen expression in the skin and is involved in the bleomycin mouse model of disease. Targeting such a pathway may be useful in the disease.

Disclosure of interest None declared

\section{P106 ABSTRACT WITHDRAWN}

\section{P107 TARGETING ACTIVATED SYNOVIAL FIBROBLASTS USING PHOTODYNAMIC THERAPY IN EXPERIMENTAL ARTHRITIS}

${ }^{1}$ DN Dorst ${ }^{*},{ }^{1} \mathrm{M}$ Rijpkema, ${ }^{2} \mathrm{M}$ Buitinga, ${ }^{3} \mathrm{M}$ Brom, ${ }^{1} \mathrm{DL}$ Bos, ${ }^{4} \mathrm{~A}$ Freimoser, ${ }^{4} \mathrm{C}$ Klein, ${ }^{1} \mathrm{~B}$ Walgreen, ${ }^{1} \mathrm{PM}$ van der Kraan, ${ }^{1} \mathrm{M}$ Gotthardt, ${ }^{1} \mathrm{Ml}$ Koenders. ${ }^{1}$ Radboud university medical centre, Nijmegen, Netherlands; ${ }^{2} \mathrm{KU}$ Leuven, Leuven, Belgium; ${ }^{3}$ Radboudumc, Leuven, Netherlands; ${ }^{4}$ Roche innovation centre, Zurich, Austria

\subsection{6/annrheumdis-2018-EWRR2018.122}

Introduction Activated synovial fibroblasts (SF) are characterised by the expression of Fibroblast Activation Protein (FAP). Here, we investigated the potential of photodynamic therapy (PDT) targeting FAP to selectively induce cell death in these 